\title{
Qualidade técnica e satisfação relacionadas às próteses totais
}

\author{
Technical quality and satisfaction \\ related to full conventional dentures
}

Anna Paula Serêjo da Costa ${ }^{1}$

Flávia Christiane de Azevedo Machado ${ }^{2}$

Anna Lepríncia Bezerra Pontes Pereira ${ }^{1}$

Adriana da Fonte Porto Carreiro ${ }^{1}$

Maria Ângela Fernandes Ferreira ${ }^{1}$

${ }^{1}$ Departamento de Odontologia, Centro de Ciências da Saúde, Universidade Federal do Rio Grande do Norte. Av. Salgado Filho 1787, Lagoa Nova. 59056-000 Natal RN. ninha_serejo@hotmail.com ${ }^{2}$ Instituto Federal de Educação, Ciência e

Tecnologia do Rio Grande do Norte (IFRN).

\begin{abstract}
Tooth loss is highly prevalent in Brazil, especially among the elderly, where more than three million need bimaxillary dentures. The federal government instituted Specialized Dental Care Centers (SDCC) to remedy this situation. The scope of this study was to evaluate one attribute of these Centers, namely the provision of full conventional dentures (FCD) from the perspective of quality and satisfaction related to this product. A cross-sectional study was conducted with 149 individuals fitted with such dentures in SDCC of Rio Grande do Norte between 2007 and 2009. Data were obtained by questionnaire and clinical examination of FCD regarding its retention, stability, aesthetics, and fixation. From the 233 dentures evaluated, 52.7\% of upper FCD and 9.5\% of lower FCD were technically satisfactory. With respect to the association between technical quality and satisfaction, the chi-square test indicated that upper FCDs were technically adequate $(p=0.041)$, particularly in terms of retention ( $p$ $=0.002)$ and stability $(p=0.000)$ and were associated with patient satisfaction. Thus, the FCD satisfied the users, despite the technical defects. However, this satisfaction may come from the fitting itself rather than its functionality.
\end{abstract}

Key words Dental prosthesis, Satisfaction of the patient and evaluation of health services
Resumo A perda dentária é muito prevalente no Brasil, sobretudo entre idosos, onde mais de 3 milhões precisam de prótese total bimaxilar. Para mudar este quadro, o governo Federal instituiu os Centros de Especialidades Odontológicas (CEO). Assim, este estudo avaliou uma das atribuições desses Centros, a provisão de próteses totais convencionais (PTC) sob a ótica da qualidade e satisfação relacionadas a este produto. Para tanto, fezse um estudo seccional com 149 indivíduos reabilitados em CEO do Rio Grande do Norte com tais próteses entre 2007 a 2009. Os dados foram obtidos por questionário e exame clínico da PTC quanto a sua retenção, estabilidade, estética e fixação. Das 233 PTC avaliadas; 52,7\% das superiores e 9,5\% das inferiores foram tecnicamente satisfatórias. Contudo, 69,1\% ( $n=103)$ dos entrevistados relataram satisfação com suas próteses. Quanto a associação entre qualidade técnica e satisfação, o teste Qui-quadrado indicou que PTC superiores tecnicamente adequadas ( $p=0,041)$, sobretudo quanto a retenção $(p=0,002)$ e estabilidade $(p=0,000)$, estavam associados à satisfação positiva do usuário. Assim, as próteses satisfizeram à população assistida, apesar dos déficits técnicos. No entanto, essa satisfação pode advir da reabilitação em si e não de sua funcionalidade.

Palavras-chave Prótese dentária, Satisfação do paciente, Avaliação de serviços de saúde 


\section{Introdução}

Apesar dos avanços da Odontologia, a perda dentária ainda é um grave problema de saúde pública, principalmente entre idosos ${ }^{1}$. No Brasil, 7 milhões de idosos necessitam de prótese total mono ou bimaxilar ${ }^{2}$. Tal fato decorre, dentre outros fatores, da deficiência de acesso à assistência odontológica de média e alta complexidades ${ }^{3}$.

Para combater a morbidade da perda dentária e de outros agravos bucais, o governo brasileiro instituiu os Centros de Especialidades Odontológicas (CEO) integrantes da Política Nacional de Saúde Bucal ${ }^{4}$. Esses Centros devem atuar de forma integrada à atenção odontológica primária, assegurando atendimento nos níveis secundários e terciários ${ }^{5}$.

Dentre suas atribuições, esta a provisão e a reabilitação com próteses dentárias totais. Essa reabilitação deve permitir o desenvolvimento satisfatório das atividades relacionadas à boca e dentes como a fonação e a mastigação, bem como oferecer conforto e uma aparência aceitável ${ }^{6}$.

Todavia, o sucesso da reabilitação não depende exclusivamente da acurácia técnica, mas de processos adaptativos individuais que interferem na aceitação da prótese pelo usuário ${ }^{7}$. Por isso, é comum a não adaptação e a insatisfação com próteses novas e tecnicamente aceitáveis ${ }^{7,8}$.

Assim, a investigação normativa (qualidade técnica) e perceptiva (satisfação) das próteses são elementos indicativos da eficácia dos serviços de saúde e pode contribuir para monitorar a qualidade desses serviços, permitindo seu aperfeiçoamento ${ }^{9,10}$.

Diante do exposto, o objetivo deste estudo é avaliar a qualidade técnica e a satisfação dos usuários em relação às próteses totais convencionais confeccionadas em Centros de Especialidades Odontológicas da mesorregião Leste Potiguar do Rio Grande do Norte.

\section{Método}

Este é um estudo transversal realizado em todos os CEO da mesorregião Leste Potiguar do RN que disponibilizam reabilitações com PTC há no mínimo 01 ano. Essa mesorregião concentra tanto o maior número de $\mathrm{CEO}$ do estado do RN como aqueles com destaque nacional segundo o Conselho Federal de Odontologia ${ }^{11}$. No entanto, só 04 dos 09 Centros desta região produzem $\mathrm{PTC}^{11}$. Esses Centros estão localizados nos municípios de Natal, Parnamirim, São Gonçalo do
Amarante e Macaíba e são classificados como tipo II (possui 4 a 6 cadeiras odontológicas), a exceção de Natal que é tipo III (mínimo de 7 cadeiras odontológicas) .

A população do estudo foi composta por indivíduos reabilitados há ao menos 1 ano nos CEO citados acima. Desta população fonte, foram selecionados intencionalmente 149 pessoas que dispunham de informações relativas a nome, endereço e/ou telefone nos arquivos dos CEO sob estudo. A quantidade de pessoas captadas foi superior ao valor obtido com os dados de estudo piloto realizado $(n=129)$, a partir de prevalência de dificuldades relacionas às PTC de $45 \%$, margem de erro de $20 \%$ e taxa de não-resposta de $10 \%$.

Todo o protocolo de estudo foi aprovado pelo comitê de ética da Universidade Federal do Rio Grande do Norte.

Os participantes foram submetidos a exame clínico e responderam a um questionário de avaliação de satisfação. A satisfação foi avaliada de duas maneiras: através de uma pergunta geral sobre a satisfação com relação às próteses confeccionadas nos CEO (se estava satisfeito ou não com as PTC) e através do questionário idealizado por Sato et al. ${ }^{12}$ e validado por Cunha ${ }^{13}$ onde a satisfação é avaliada em relação à mastigação, gustação, pronúncia, dor, estética, adaptação, retenção e conforto proporcionados pelas PTC.

O exame clínico visava captar informações a respeito da presença/ ausência de lesões orais possivelmente associadas ao uso das próteses e presença/ausência de biofilme dentário. Essas observações foram feitas para avaliar a fixação das próteses, já que o protocolo de exame técnico das próteses seguiu as diretrizes do SB Brasil $2010^{14}$.

Assim, as próteses foram examinadas quanto à retenção (se estava apertada ou folgada). A prótese deveria manter-se em posição durante os movimentos fisiológicos normais do paciente como: deglutição, mastigação, fonação. Estabilidade, uma vez em posição, não devia deslocar-se ou mover-se, seja em repouso, seja em movimento. Gentilmente, foi forçado pelo examinador a base da prótese na direção dos tecidos e verificava-se se deslocava ou não, fixação (ocasionava ou não lesão na boca) e estética (se estava adequada ou não ao perfil facial do paciente, detinha ou não manchas e fraturas). A adequação técnica das PTC era confirmada se todas as características estivessem a contento ${ }^{14}$.

As variáveis relativas à satisfação e qualidade técnica associadas às próteses sob estudo foram apresentadas de maneira descritiva por meio de 
números absolutos e proporções. Já a averiguação da existência de associação entre essas variáveis foi feita pelo teste Qui-quadrado e exato de Fisher, com nível de significância de 5\%.

\section{Resultados}

Participaram desta pesquisa 149 indivíduos de um universo de 1882 pessoas que possuíam dados de identificação disponíveis. Houve três perdas devido a falecimento, duas perdas das próteses e uma recusa para participação.

Nos quatro Centros de Especialidades estudados predominaram usuários do sexo feminino ( $\mathrm{n}=111 ; 74,5 \%)$, com idade média de 59,48 anos (36-89) e renda familiar média de 540 reais (0-3000).

Grande parte dos indivíduos já tinha experiência com uso de próteses, sendo $86,6 \%$ ( $\mathrm{n}=$ 129) com prótese superior há 20 anos (0-30 anos) e $51,7 \%(\mathrm{n}=77)$ com prótese inferior há 10,75 anos (0-20 anos).

Em relação à satisfação, 69,1\% $(\mathrm{n}=103)$ dos indivíduos dos indivíduos responderam estar satisfeitos com suas próteses. No entanto, houve considerável insatisfação quanto à retenção e conforto da prótese inferior (ver Figura 1).

Ao exame clínico 47,3\% das PTC superiores e $90,5 \%$ das inferiores estavam tecnicamente insatisfatórias. As superiores, sobretudo, quanto à estabilidade e fixação e as inferiores, pela estética e fixação (Figura 2).

Após realização dos testes Qui-quadrado, houve associação entre a qualidade técnica satis- fatória das PTC superiores e satisfação do usuário $(\mathrm{p}=0,041)$. Ao especificar os critérios técnicos, a associação permaneceu entre satisfação do indivíduo e retenção $(p=0,002)$ e satisfação do indivíduo e estabilidade $(\mathrm{p}<0,001)$ (Tabela 1).

\section{Discussão}

O edentulismo é uma condição crônica ${ }^{15}$ que pode ocasionar dificuldades sociais, psicológicas e físicas (mastigação, digestão, fala, atrofia das estruturas alveolares, diminuição do tônus muscular facial) ${ }^{16,17}$. Portanto, a reabilitação deve proporcionar não apenas a função dentária e/ou bucal, mas também a reintegração desse indivíduo ao convívio social.

Os indicadores de necessidade de prótese dos levantamentos epidemiológicos SB Brasil 2003 e 2010 revelam uma grande demanda reprimida desta reabilitação, sobretudo entre idosos. A necessidade de reabilitação surge na adolescência e se acumula ao longo da vida do indivíduo (SB 2003), sobretudo naqueles pertencentes às classes socais mais baixas ${ }^{1}$.

De fato, neste estudo, a população assistida nos CEO compunha-se de adultos e idosos com renda familiar em torno de 510 reais. Houve maior frequência de mulheres uma vez que estas procuram mais os serviços de saúde pública, seja para cuidar de seus filhos ou delas próprias ${ }^{3}$. No tocante à reabilitação protética, é mais compreensível, na medida em que se deseja não só o resgate da função mastigatória, mas também o restabelecimento da harmonia facial ${ }^{18,19}$.
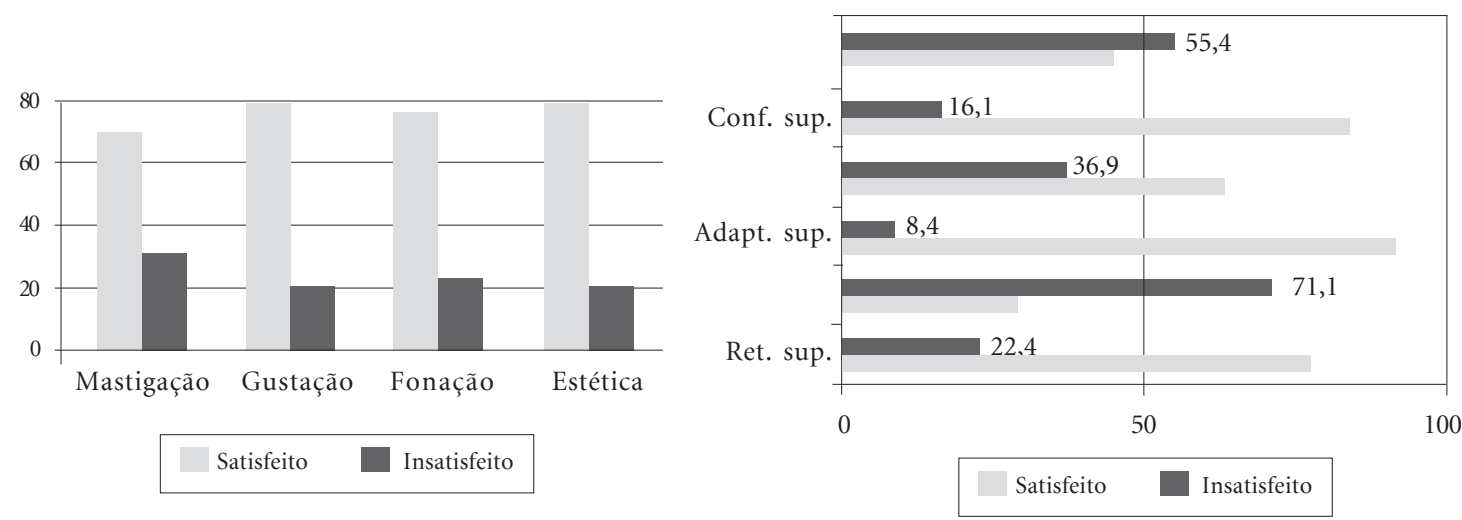

Figura 1. Percentual de usuários satisfeitos com as próteses quanto à estética, capacidade de mastigar, saborear os alimentos, falar, conforto, adaptação e retenção. 


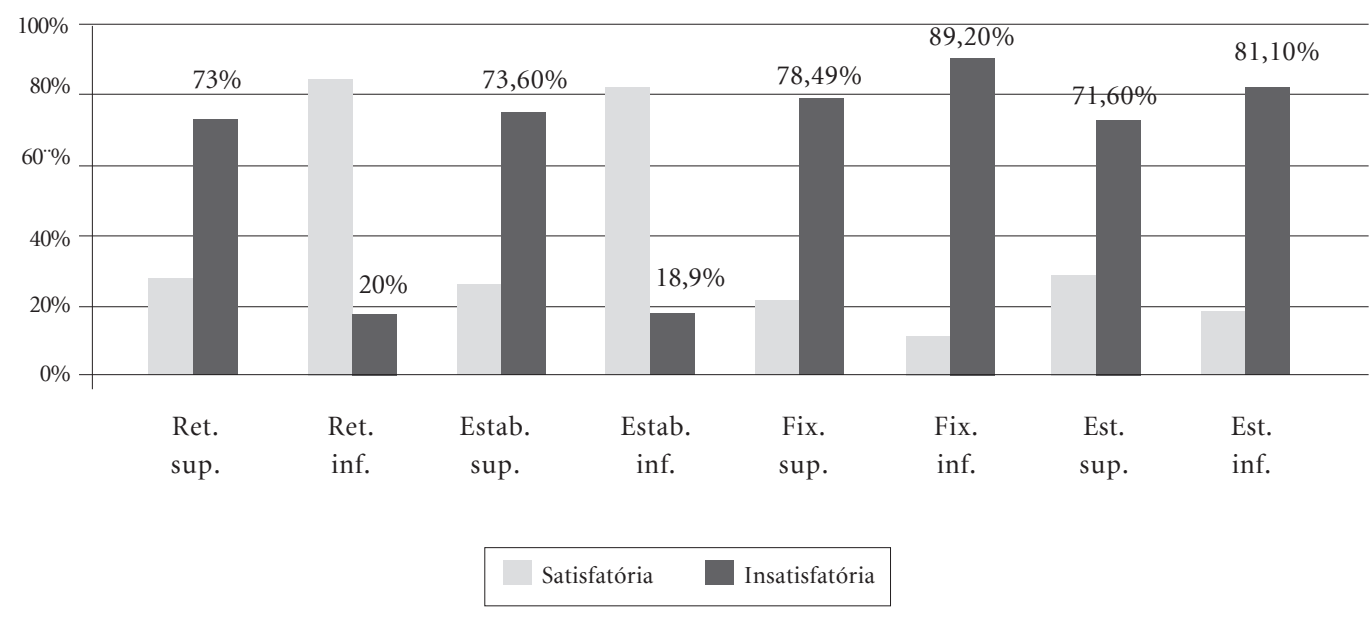

Figura 2. Qualidade técnica em relação a cada item de avaliação das próteses totais convencionais confeccionadas nos CEO da Grande Natal entre 2007 e 2009.

Tabela 1. Relação entre a satisfação geral associada às próteses superiores sob estudo com as características técnicas das mesmas.

\begin{tabular}{|c|c|c|c|c|c|c|c|}
\hline \multirow{3}{*}{ Variáveis } & \multicolumn{4}{|c|}{ Satisfação geral } & \multirow{3}{*}{ OR } & \multirow{3}{*}{ OR(IC 95\%) } & \multirow{3}{*}{$\mathbf{P}$} \\
\hline & \multicolumn{2}{|c|}{ Sim } & \multicolumn{2}{|c|}{ Não } & & & \\
\hline & $\mathbf{N}$ & $\%$ & $\mathbf{N}$ & $\%$ & & & \\
\hline \multicolumn{8}{|l|}{ Qualidade técnica da prótese superior } \\
\hline Satisfatória & 60 & $76,9 \%$ & 18 & $23,1 \%$ & 2,093 & $1,025-4,272$ & 0,041 \\
\hline Insatisfatória & 43 & $61,4 \%$ & 27 & $38,6 \%$ & & & \\
\hline \multicolumn{8}{|l|}{ Retenção da prótese superior } \\
\hline Folgada & 20 & $50 \%$ & 20 & $50 \%$ & 0,301 & $0,140-0,647$ & 0,002 \\
\hline Apertada & 83 & $76,9 \%$ & 25 & $23,1 \%$ & & & \\
\hline \multicolumn{8}{|l|}{ Estabilidade da prótese superior } \\
\hline Presença de deslocamento e báscula & 17 & $43,6 \%$ & 22 & $56,4 \%$ & 0,207 & $0,094-0,452$ & $<0,001$ \\
\hline Ausência de deslocamento e báscula & 86 & $76,9 \%$ & 23 & $21,1 \%$ & & & \\
\hline \multicolumn{8}{|l|}{ Fixação da prótese superior } \\
\hline Apresentam lesão & 21 & $65,6 \%$ & 11 & $34,4 \%$ & 0,792 & $0,345-1,819$ & 0,665 \\
\hline Não apresentam lesão & 82 & $70,7 \%$ & 34 & $29,3 \%$ & & & \\
\hline \multicolumn{8}{|l|}{ Estética da prótese superior } \\
\hline $\begin{array}{l}\text { Presença de manchas e/ou fraturas e/ou } \\
\text { desconformidade com o perfil facial }\end{array}$ & 32 & $76,2 \%$ & 10 & $23,8 \%$ & 1,577 & $0,697-3,572$ & 0,325 \\
\hline $\begin{array}{l}\text { Ausência de manchas, fraturas e } \\
\text { desconformidade com o perfil facial }\end{array}$ & 71 & $67,0 \%$ & 35 & $33,0 \%$ & & & \\
\hline
\end{tabular}

Para assegurar a efetividade dos serviços de saúde é necessário captar informações para propiciar decisões mais adequadas e ações resolutivas, ou seja, é preciso avaliar esses serviços. Alguns autores afirmam a necessidade de se institucionalizar a avaliação do serviço como rotina em diversos setores da saúde através da formulação de uma Política Nacional de Avaliação em Saúde com o intuito de possibilitar o aprimoramento institucional e profissional ${ }^{20-22}$.

Foi com essa perspectiva, que a qualidade técnica e a satisfação dos usuários relativas às pró- 
teses dentárias confeccionadas nos CEO foram avaliadas neste estudo. A qualidade técnica deve ser definida em relação a fatores objetivos, sobretudo, quanto à retenção e à estabilidade ${ }^{7,18,23}$. Entretanto, a estética, a adaptação, a presença de lesão na mucosa oral e características clínicas da cavidade oral também podem ser aferidas ${ }^{23}$. Todavia, todos esses fatores são de difícil avaliação, devido à dificuldade de definir requisitos em termos de qualidade. Assim, a avaliação desses fatores tende a ser subjetiva, mostrando grande variabilidade, dificultando a comparação entre os estudos ${ }^{24}$.

Este estudo utilizou o critério da retenção, estabilidade, fixação e estética para avaliação normativa das próteses chegando a resultados sensivelmente satisfatórios. A maioria das próteses totais convencionais superiores foram tecnicamente satisfatórias, porém a maioria das inferiores foi insatisfatória. Esse resultado pode está relacionado às características clínicas dos indivíduos, visto que características como altura e conformação do rebordo e tipo de fibromucosa podem comprometer significativamente os itens de retenção e estabilidade ${ }^{25}$. Os participantes do estudo tinham histórico precoce de edentulismo, aumentando a probabilidade de altura e conformação de rebordos inadequados para uma retenção e estabilidade satisfatórias das PTC. De fato, retenção e estabilidade foram itens com grande deficiência normativa.

Em relação à satisfação dos usuários com as PTC, apesar das deficiências funcionais destes dispositivos, a maioria dos pacientes encontrava-se satisfeita em consonância com outros estudos que mostram a variação média de satisfação em torno de $50 \%$ a $75 \%{ }^{26,27}$. Segundo estudo de Berg ${ }^{24}$, a satisfação é influenciada por fatores como as expectativas dos pacientes, qualidade técnica das próteses, características clínicas do indivíduo, experiência anterior com o uso de prótese e a personalidade do paciente.

Neste estudo, uma associação significativa foi encontrada entre a qualidade técnica da prótese total convencional superior e a satisfação dos usuários, confirmando que esses conseguem perceber os problemas presentes em suas próteses superiores em conformidade com estudos anteriores $^{28-31}$.

A retenção e a estabilidade da prótese superior foram as variáveis da qualidade técnica que influenciaram na satisfação dos usuários com relação ao uso da prótese. O que coincide com os achados de outros estudos que relatam a forte associação do uso da prótese com as variáveis que garantem a possibilidade de desenvolver a mastigação e a fonação adequadas ${ }^{7,18,23}$. Alguns autores referem ainda à questão da estética ${ }^{23}$, bem como a opinião de outras pessoas ${ }^{32}$ e o desconforto $^{8,18,33}$ como fatores que influenciam na satisfação dos usuários, contudo este estudo não encontrou associação significativa com essas variáveis assim como outros ${ }^{34}$.

Com relação às próteses totais inferiores não foram encontradas associações com a satisfação neste estudo, provavelmente, devido à menor quantidade de próteses analisadas em relação às superiores, como também devido à conscientização dos pacientes sobre as limitações dessas próteses em virtude de suas características clínicas individuais como altura e conformação do rebordo mandibular.

$\mathrm{O}$ uso anterior de próteses totais não teve nenhuma associação com a satisfação. Berg $^{24} \mathrm{e}$ Pinelli ${ }^{30}$ obtiveram o mesmo resultado, enquanto Weinstein et al. ${ }^{35}$, e van Waas ${ }^{28}$ encontraram uma fraca associação, demonstrando que os pacientes que nunca usaram próteses estavam menos satisfeitos com estas do que os que haviam usados mais de um conjunto.

A influência da idade do paciente na satisfação dos usuários foi explorada em vários estudos, alguns demonstraram que pacientes de mais de sessenta anos tiveram mais dificuldades de se adaptar às próteses totais convencionais e a usavam por menos tempo quando comparados àqueles com idade inferior ${ }^{15,30,36}$. Devido ao próprio processo de envelhecimento, possuem maior dificuldade de adaptação decorrente da redução nas suas habilidades de estabelecer novos arcos reflexos sensoriais ${ }^{36}$. Entretanto, de acordo com a maioria dos estudos encontrados na literatura e no presente estudo não foi observado relação entre a idade e a satisfação $0^{23,24,28,37-39}$.

Os fatores sociais e demográficos como sexo, estado civil, renda familiar e ocupação também não apresentaram associação com a satisfação dos usuários com relação às próteses totais convencionais, o que coincide com outras investigações presentes na literatura ${ }^{25,26}$. Berg et al. ${ }^{32}$ investigaram além desses fatores a atividade social, condições de moradia, relação com vizinhos e opinião de outras pessoas, mas apenas o último fator influenciou na aceitação das próteses.

Para finalizar esta discussão, enfatizamos que para avaliar um serviço, seja em relação aos seus aspectos mais gerais ou específicos, devem-se propor alternativas para sua melhoria. Um fato bastante preocupante encontrado neste estudo foi que $18 \%$ das pessoas não usavam mais as 
próteses, principalmente por problemas de retenção e estabilidade da PTC inferior, apesar das próteses apresentarem-se com no máximo 3 anos de uso. Isso reforça a hipótese do alto contingente de PTC insatisfatórias advir de um prognóstico desfavorável. Assim, é preciso rever os critérios para indicar a reabilitação com PTC, evitando dispêndio de recursos públicos. Neste sentido, as próteses implanto-suportadas ou o fornecimento de materiais adesivos para a fixação seria uma alternativa viável ${ }^{40}$. De fato, a reabilitação com implantes foi englobada recentemente pela Política Nacional de Saúde Bucal Brasil Sorridente reiterando a hipótese acima.

\section{Colaboradores}

APS Costa trabalhou na concepção, no delineamento, na análise e interpretação dos dados, na redação do artigo e na aprovação da versão a ser publicada; FCA Machado trabalhou no delineamento, interpretação dos dados e na redação da versão a ser publicada; ALBP Pereira trabalhou na concepção, na análise dos dados e na aprovação da versão a ser publicada; AFP Carreiro trabalhou na concepção, no delineamento e na aprovação da versão a ser publicada; MAF Ferreira trabalhou na concepção, no delineamento, na análise e interpretação dos dados, na redação do artigo e na aprovação da versão a ser publicada.

\section{Conclusão}

Os Centros de Especialidades estão confeccionando próteses de qualidade técnica insatisfatória, sobretudo, quanto à retenção. Entretanto, é necessário avaliar se tal resultado advém do prognóstico desfavorável em reabilitar com próteses convencionais indivíduos com histórico precoce de perda dentária.

Todavia, constata-se que os CEO estão avançando quanto à redução das iniquidades em saúde bucal ao assistir à população que concentra as maiores demandas de reabilitação protética. No entanto, para continuar avançando, se faz necessário instituir a avaliação periódica de suas ações através de indicadores normativos e perceptivos que poderão aferir sua efetividade. 


\section{Referências}

1. Brasil. Ministério da Saúde (MS). Projeto SB Brasil 2003: condições de saúde bucal da população brasileira 2002-2003. Resultados principais. Brasília: MS; 2004.

2. Brasil. Ministério da Saúde. Projeto SB Brasil 2010. Resultados parciais. [acessado 2011 fev 10]. Disponível em: http://189.28.128.100/dab/docs/geral/ apresentacao_SB2010.pdf

3. Instituto Brasileiro de Geografia e Estatística (IBGE). Acesso e utilização de serviços de saúde 2003. Pesquisa Nacional por Amostra de Domicílios. Ministério do Planejamento, Orçamento e Gestão. Rio de Janeiro: IBGE, CDDI; 2005.

4. Brasil. Ministério da Saúde. Portaria no. 599 de 2006. Define a implantação de Especialidades Odontológicas (CEO) e de Laboratórios Regionais de Próteses Dentárias (LRPDs) e estabelecer critérios, normas e requisitos para seu credenciamento. Diário Oficial da União 2006; 24 mar.

5. Brasil. Ministério da Saúde. Diretrizes da Política Nacional de Saúde Bucal. Brasília: MS; 2004.

6. Gennari Filho H. O exame clínico em prótese total. Revista Odontológica de Araçatuba 2004; 25(2):62-71.

7. Leles CR, Nakaoka MM, Souza RF, Compagnoni MA. Estudo Restrospectivo dos Fatores Associados à Longevidade de Próteses Totais. Parte I - Avaliação Subjetiva e Queixas dos Pacientes. Rev Fac Odontol 1999; 2(1):61-66.

8. Brunello DC, Mandikos, MN. Construction faults, age, gender, and relative medical health: factors associates with complaints in complete denture patients. J Prosthet Dent 1998; 79(5):545-554.

9. Gattinara BC, Ibacache J, Puente C, Giaconi J, Caprara A. Percepción de la comunidad acerca de la calidad de los servicios de salud públicos en los distritos Norte e Ichilo, Bolivia. Cad Saude Publica 1995; 11(3):425-438.

10. Moris AJ, Burke FJT. Primary and secondary dental care: how ideal is the interface? Br Dent J 2001; 191(12):666-670

11. Conselho Regional de Odontologia do Rio Grande do Norte. Canguaretama e Macaíba vão representar o RN no Prêmio Brasil Sorridente 2010. [acessado 2011 out 19]. Disponível em: http://www.crorn. org.br/noticias/ver/485

12. Sato Y, Hamada S, Akagawa Y, Tsuga K. A method for quantifying overall satisfaction of complete dentures patients. J Oral Rehabil 2000; 27(11):952-957.

13. Cunha EFS. Avaliação de prótese total bimaxilar em função das características da área basal [tese]. São Paulo: Universidade de São Paulo; 2004.

14. Brasil. Ministério da Saúde (MS). Manual do pesquisador de campo. Projeto SB Brasil 2010. Brasília: MS; 2009.

15. Awad MA, Feine JS. Measuring patient satisfaction with mandibular protheses. Community Dent Oral Epidemiol 1998; 26(6):400-405.

16. Tallgren A, Lang BR, Miller RL. Longitudinal Study of soft-tissue profile changes in patients receiving immediate complete dentures. Int I Prosthodont 1991; 4(1):9-16.
17. Fiske J, Davis DM, Frances C, Gelbier S. The emotional effects of tooth loss in edentulous people. $\mathrm{Br}$ Dent J 1998; 184(2):90-93.

18. Langer A, Michman J, Seifert I. Factors influencing satisfaction with complete dentures in geriatric patients. J Prosthet Dent 1961; 11:1019-1031.

19. Gordon SR, Fryer GE, Niessen L. Patient satisfaction with current dental condition related to selfconcept and dental status. J Prosthet Dent 1988; $59(3): 323-327$

20. Contrandiopoulos AP. Avaliando a institucionalização da avaliação. Cien Saude Colet 2006; 11(3):705711.

21. Felisberto E. Da teoria a formulação de uma política nacional de avaliação em saúde: reabrindo o debate. Cien Saude Colet 2006; 11(3):553-563.

22. Hartz ZMA. Institucionalizar e qualificar a avaliação: outros desafios para a atenção básica. Cien Saude Colet 2002; 7(3):419-421.

23. Carlsson GE, Otterland A, Wennstrom. A patient factors in appreciation of complete dentures. J Prosthet Dent 1967; 17(4):322-328.

24. Berg E. Acceptance of full dentures. Int Dent J 1993; 43:299-306.

25. Seifert I, Langer A, Michmann J. Evaluation of physicologic factors in geriatric denture patients. J Prosthet Dent 1962; 12:516-523.

26. Turker SB, Sener ID, Ozkan YK. Satisfaction of the complete denture wearers related to various factors. Arch Gerontol Geriatr 2009; 49(2):126-129.

27. Celebic A, Kenezovic-Zlataric D. A comparison of patient's satisfaction between complete and partial removable denture wearers. J Dent 2003; 31(7):445451.

28. Van Waas MAJ. The influence of clinical variables on patients satisfaction with complete dentures. $J$ Prosthet Dent 1990; 63(3):307-310.

29. Kalk W, de Baat C, Kaandorp AJG. Comparison of patients views and dentists evaluation 5 years after complete denture treatment. Communit Dent Oral Epidemiol 1991; 19(4):213-216.

30. Pinelli LAP. Avaliação do grau de satisfação e qualidade das próteses em pacientes portadores de dentaduras. [tese] Araraquara: Universidade Estadual Paulista; 2001.

31. Fenlon MR, Sherriff M. Na investigation of factors influencing patients satisfaction with new complete dentures using strutural equation modelling. J Dent 2008; 36(6):427-434.

32. Berg E, Johnson TB, Ingebretsen R. Social variables and patients acceptance of complete dentures. Acta Odontolol Scand 1985; 43(4):199-203.

33. Sheippard IM, Schwartz LR, Sheppard SM. Survey of the oral status of complete denture patients. $J$ Prosthet Dent 1972; 28(2):121-126.

34. Smith PW, McCord JF. What do patients expect from complete dentures? J Dent 2004; 32(1):3-7.

35. Weinstein M, Schuchman J, Lieberman J, Rosen P. Age and denture experience as determinants in patient denture satisfaction. J Prosthet Dent 1988; 59(3): 327-329. 
36. Powter G, Cleaton-Jones P. Quantitative assessment of some factors governing complete dentures sucess. J Dent Assoc S Afr 1980; 35(1):5-8.

37. Kawai Y, Murakami H, Shariati B, Klemetti E, Blomfield JV, Billette L, Lund JP, Feine JS. Do traditional tecniques produce better conventional dentures than simplifield techniques? J Dent 2005; 33(8):659-668.

38. Critchlow SB, Ellis JS. Prognostic indicator for conventional complete denture therapy: A review of the literature. J Dent 2010; 38(1):2-9.

39. Ellis JS, Pelekis ND, Thomason JM. Conventional rehabilitation of edentulous patients: the impact on oral health related quality of life and patient satisfaction. J Prosthodont 2007; 16(1):37-42.

40. Roumanas ED. The social solution - denture esthetics, phonetics and function. J Prosthodont 2009; 18(2):112-115.

Artigo apresentado em 31/11/2011

Aprovado em 07/12/2011

Versão final aprovada em 19/01/2012 